\title{
Repelência e atividade inseticida de pós vegetais sobre Zabrotes subfasciatus Boheman em feijão-fava armazenado
}

GIRÃO FILHO, J.E. **; ALCÂNTARA NETO, F.²; PÁDUA, L.E.M.'2; PESSOA, E. F'.

${ }^{1}$ Programa de Pós-Graduação em Agronomia, Mestrado, Universidade Federal do Piauí, Rua Dirce de Oliveira, 3597 - Campus da Socopo - Cep: 64049-550 - Teresina-PI, * giraofilho@hotmail.com; ${ }^{2}$ Universidade Federal do Piauí, Centro de Ciências Agrárias, Departamento de Fitotecnia, Campus da Socopo - Cep: 64049-550 Teresina-PI.

RESUMO: O objetivo desta pesquisa foi avaliar o potencial inseticida de Piper nigrum L. (pimenta do reino), Ruta graveolens L. (arruda), Laurus nobilis L. (louro), Syzygium aromaticum (L.) Merr. \& L.M.Perry (cravo da índia), Chenopodium ambrosioides L. (mastruz), Piper tuberculatum Jaqc. (pimenta de macaco), Tagetes erecta L. (cravo de defunto), Cymbopogon nardus L. (citronela) e Melissa officinalis L. (erva cidreira) sobre Z. subfasciatus Boh. através dos testes de confinamento, onde os insetos foram confinados durante cinco dias em um recipiente contendo dez grãos de feijão-fava com $0,3 \mathrm{~g}$ do pó da planta a ser testada, e, com chance de escolha por meio de uma arena circular, os insetos tiveram a possibilidade de escolha entre grãos com os pós e a testemunha, grãos sem pó das plantas. Observou-se que houve plantas que atuaram como inseticida, outras que repeliram o inseto e não causaram a morte, e outras, que além de repelir também mataram os insetos quando em contato (cravo da índia e matruz). Concluímos que: P. nigrum, P. tuberculatum, S. aromaticum e C. ambrosioides são tóxicas à Z. subfasciatus causando-lhes a morte, L. nobilis, T. erecta, e C. nardus não apresentaram efeito tóxico sobre Z. subfasciatus, C. ambrosioides, S. aromaticum, e C. nardus são repelentes à Z. subfasciatus; $P$. nigrum, $P$. tuberculatum, e T. erecta são neutras, e que $M$. officinalis não apresentou nenhum efeito sobre Z. subfasciatus em relação aos parâmetros avaliados.

Palavras-chave: plantas inseticidas, manejo alternativo, caruncho, Phaseolus lunatus

ABSTRACT: Repellency and insecticidal activity of plant powders on Zabrotes subfasciatus (Boh.) on stored lima beans. The purpose of this research was to evaluate the insecticidal potential of Piper nigrum L., Ruta graveolens L., Laurus nobilis L., Syzygium aromaticum Merr. \& L.M.Perry., Chenopodium ambrosioides L., Piper tuberculatum Jaqc., Tagetes erecta L., Cymbopogon nardus $\mathrm{L}$. and Melissa officinalis $\mathrm{L}$. against Zabrotes subfasciatus by free choice, in which, by means of a circular arena, insects had a choice, treatments with or without powder and no choice tests, where insects were confined for five days in a container containing ten grains of lima beans with $0.3 \mathrm{~g}$ of powder to be tested. It was observed that some plants have killed the insect while others just repelled the insect, causing no harm to it. Also, one of the studied plants repelled and killed the insects (S. aromaticum, C. ambrosioides and $P$. tuberculatum). We can conclude that: $P$. nigrum, $P$. tuberculatum, $S$. aromaticum e $C$. ambrosioides are toxic to the $Z$. subfasciatus, causing its death. L. nobilis, T. erecta e $C$. nardus do not present any toxic effect over Z. subfasciatus. C. ambrosioides, S. aromaticum e C. nardus are repellent to Z. subfasciatus. P. nigrum, P. tuberculatum e T. erecta are neutral and $M$. officinalis have presented no effects over $Z$. subfasciatus in relation to the parameters evaluated.

Keywords: insecticidal plants, alternative management, weevil, Phaseolus lunatus

\section{INTRODUÇÃO}

O gorgulho Zabrotes subfasciatus é a principal praga dos grãos ou sementes de feijãofava (Phaseolus lunatus Linnaeus) durante o armazenamento, causando-Ihes danos qualitativos e quantitativos. O controle químico se constitui na principal estratégia de manejo das pragas de grãos armazenados e, seu uso frequente tem provocado o surgimento de populações de insetos resistentes

Recebido para publicação em 13/09/2013

Aceito para publicação em 11/03/2014

10.1590/1983-084X/13_087

Rev. Bras. PI. Med., Campinas, v.16, n.3, p.499-504, 2014. 
às principais classes de inseticidas utilizados para tal fim, tais como piretróides e organofosforados (Silva et al., 2013; Ribeiro et al., 2003; Beckel et al., 2002; Beckel et al., 2005), sendo um grande problema, tanto para os agricultores, quanto para as indústrias que necessitam desenvolver novos sítios alvos (Perry et al., 2011).

Estudos com plantas inseticidas têm aumentado nos últimos anos, entretanto, Procópio et al. (2003a) relatam que as pesquisas ainda são incipientes comparativamente ao grande potencial botânico disponível na natureza. Além disso, a eficiência desses inseticidas naturais varia bastante de acordo com a espécie de inseto a ser controlada, espécie e parte da planta utilizada, a forma de aplicação, e até mesmo local de coleta da planta (Oliveira \& Vendramim, 1999; Procópio et al., 2003a; Procópio et al., 2003b; Mazzonetto \& Vendramim, 2003; Paranhos et al., 2005).

Os inseticidas botânicos são geralmente usados na forma de pós, extratos, ou óleos. Segundo Procópio et al. (2003a), a forma de pós é a mais preferida pelos agricultores devido à facilidade de aplicação e pela natureza do material que se deseja proteger, no caso, grãos ou sementes.

Objetivou-se com esta pesquisa avaliar o potencial inseticida de Piper nigrum (pimenta do reino), Ruta graveolens (arruda), Laurus nobilis (louro), Syzygium aromaticum (cravo da índia), Chenopodium ambrosioides (mastruz), Piper tuberculatum (pimenta de macaco), Tagetes erecta (cravo de defunto), Cymbopogon nardus (citronela), e Melissa officinalis (erva cidreira) sobre Z. subfasciatus através dos testes de confinamento com chance de escolha visando encontrar uma forma de controle alternativo deste inseto.

\section{MATERIAL E MÉTODO}

Foram realizados testes de confinamento e livre chance de escolha, no período de agosto de 2012 a janeiro de 2013, no Laboratório de Fitossanidade da Universidade Federal do Piauí (UFPI), sob condições controladas de luz e temperatura, sendo; o teste de confinamento realizado com foto-fase de 12 horas, e o teste com livre chance de escolha em escuro, ambos a $28^{\circ} \mathrm{C}$ \pm 1 .

Os pós-vegetais utilizados foram obtidos a partir de frutos de pimenta do reino (Piper nigrum), folhas e talos de arruda (Ruta graveolens), folhas de louro (Laurus nobilis) e inflorescências de cravo da Índia (Syzygium aromaticum), adquiridos no mercado local de Teresina. Folhas, flores e frutos de mastruz (Chenopodium ambrosioides), frutos de pimenta de macaco (Piper tuberculatum), folhas, talos e flores de cravo de defunto (Tagetes erecta), folhas e inflorescência de citronela (Cymbopogon nardus) e folhas e talos de erva cidreira (Melissa officinalis), coletadas no Núcleo de Plantas Aromáticas e Medicinais da UFPI.

Após a aquisição, os materiais foram secos em estufa a $40{ }^{\circ} \mathrm{C}$ por 72 horas, triturados em moinho de facas e, posteriormente, em moinho de bolas por cinco minutos para obtenção do pó. Os grãos utilizados nos testes (feijão-fava boca de moça creme) foram adquiridos no mercado local de Teresina-PI. Para observação do poder inseticida dos pós-vegetais em questão, utilizaramse o teste de confinamento em delineamento inteiramente casualizado com dez tratamentos e dez repetições. As parcelas dos ensaios consistiram em um recipiente plástico com capacidade de 100 $\mathrm{mL}$, com tampa perfurada, contendo $0,3 \mathrm{~g}$ do pó vegetal a ser testado (tratamentos; testemunha sem pó), mais 10 grãos de feijão-fava e três casais de Z. subfasciatus com 0-24 horas de emergidos, os quais permaneceram confinados durante cinco dias (Procópio et al., 2003a; Mazzonetto \& Vendramim 2003). Decorrido este período os insetos foram retirados, quantificados e classificados em vivos e mortos. Quinze dias após a infestação foram quantificados os ovos viáveis e inviáveis. Foram considerados ovos viáveis aqueles com coloração esbranquiçada ou leitosa, cuja larva já estivesse penetrada no grão ou ainda dentro do ovo. Decorridos 20 dias após a infestação as observações passaram a ser diárias a fim de quantificar e retirar os insetos emergidos para cálculo do período médio de desenvolvimento (PMD), realizado através da fórmula:

$\mathrm{PMD}=\frac{\sum \text { dias de emergência } \mathrm{x} \text { insetos emergidos }}{\sum \text { insetos emergidos }}$

Os parâmetros avaliados neste teste foram: insetos mortos ao quinto dia, número de ovos viáveis, número de ovos inviáveis, insetos emergidos, mortalidade prematura ( número de ovos viáveis-insetos emergidos) e PMD.

A repelência dos pós vegetais foi observada utilizando arenas circulares constituída por um recipiente central com $10 \mathrm{~cm}$ de diâmetro por cinco centímetros de altura e oito periféricos com cinco centímetros de diâmetro por seis centímetros de altura, equidistantes e interligados ao central por meios de tubos plásticos de $12 \mathrm{~cm}$, confeccionado com mangueira de três milímetros de diâmetro. Em cada compartimento periférico sorteou-se o tratamento, $0,3 \mathrm{~g}$ do pó vegetal, inclusive a testemunha (sem pó), a ser utilizado, que foram os mesmos utilizados no teste de confinamento, exceto o pó de folhas de louro e arruda, pois não apresentaram resultados satisfatórios no primeiro 
teste. Utilizaram-se sete arenas, constituindo as repetições do ensaio em delineamento inteiramente casualizado. No recipiente central, foram soltos 40 casais de Z. subfasciatus (proporção de cinco casais por tratamento) de 0 a 48 horas de idade. Após 24 horas da liberação dos insetos no compartimento central, as tubulações de acesso aos compartimentos periféricos foram fechadas com algodão e os insetos que escolheram um determinado recipiente (tratamento) foram quantificados e descartados.

Avaliou-se neste ensaio: número de insetos atraídos e o índice de repelência (IR) onde: $I R=2 G /$ $(G+P)$ onde IR = índice de repelência, $G=\%$ insetos atraídos por tratamento e $\mathrm{P}=\%$ de insetos atraídos na testemunha. Os valores de IR variaram entre zero e dois, indicando: $I R=1$, neutra; $I R>1$, atraente e IR $<1$, repelente. Como margem de segurança para essa classificação, o erro padrão (EP) de cada tratamento foi adicionado/subtraído do valor 1,00 (indicativo de neutralidade). Assim, cada tratamento só foi considerado repelente ou atraente quando o IR estava fora do intervalo 1,00 \pm DP (Mazzonetto \& Vendramim, 2003).

Para ambos os testes, os dados, por não apresentarem normalidade segundo Shapiro-Wilk, foram submetidos à análise de variância, analisados pelo teste de Kruskal Wallis e as médias comparadas entre si pelo teste Student-Newman-Keuls (SNK) a $5 \%$ de probabilidade.

\section{RESULTADO E DISCUSSÃO}

Pôde-se observar que no teste de confinamento os pós-vegetais que tiveram maior efeito sobre a morte dos gorgulhos foram aqueles oriundos da pimenta do reino e pimenta de macaco com $100 \%$ de adultos mortos ao quinto dia, seguido por cravo da índia com $96 \%$ e matruz com $76 \%$, os quais não diferiram estatisticamente entre si pelo teste SNK, no entanto, diferindo dos demais pós estudados, os quais se igualaram estatisticamente à testemunha, não demonstrando letalidade sobre adultos de $Z$. subfasciatus (Tabela 1 ).

Em relação à pimenta do reino, resultados semelhantes foram observados por Garcia et al. (2000) onde constataram que doses de $4 \mathrm{~g} \cdot \mathrm{kg}^{-1}$ obtiveram controle absoluto do gorgulho durante oito meses de armazenamento de feijão comum com menores perdas na germinação e plântulas anormais. Oliveira \& Vendramim (1999), apesar de não terem verificado efeito repelente do pó de pimenta do reino, afirmam que o mesmo é uma fonte promissora de inseticida botânico, fato confirmado nesta pesquisa (Tabela 1; Tabela 2). Almeida et al. (2004), também verificaram mortalidade de 100\% de Callosobruchus maculatus expostos ao extrato de pimenta do reino.

A pimenta de macaco, apesar de não ter sido muito estudada em relação ao controle de pragas, vem se mostrando eficiente no controle de C. maculatus (Castro et al., 2010), Spodoptera frugiperda (Castro et al., 2008), Alabama argillacea (Miranda et al., 2002), Diatraea saccharalis (Seberón et al., 2006) com resultados semelhantes aos apresentados neste trabalho em relação ao seu potencial inseticida, sendo uma fonte promissora de substâncias químicas que podem ser utilizadas no manejo integrado de pragas, sobretudo em desenvolvimento de novas moléculas sintéticas de uso comercial.

Tabela 1. Efeito tóxico médio ( \pm EP) de pós-vegetais sobre Z. subfasciatus em teste de confinamento.

\begin{tabular}{|c|c|c|c|c|c|}
\hline Tratamentos & $\begin{array}{l}\text { Insetos } \\
\text { mortos }\end{array}$ & $\begin{array}{l}\text { Ovos } \\
\text { viáveis }\end{array}$ & $\begin{array}{l}\text { Ovos } \\
\text { inviáveis }\end{array}$ & $\begin{array}{l}\% \text { Morte } \\
\text { prematura }\end{array}$ & $\mathrm{PMD}^{* *}$ \\
\hline Cymbopogon nardus (citronela) & $0,9 \pm 1,37 a$ & $47,4 \pm 3,04 a$ & $15,1 \pm 2,5 \mathrm{a}$ & 36,5 a & 33,4 a \\
\hline Syzygium aromaticum (cravo da índia) & $5,8 \pm 0,16 b$ & $0,0 \pm 0,0 \mathrm{~b}$ & $0,4 \pm 2,0 \mathrm{~b}$ & $100,0 \mathrm{~b}$ & 0,0 * \\
\hline Ruta graveolens (arruda) & $0,3 \pm 1,27 a$ & $45,5 \pm 2,55 a$ & $10,1 \pm 1,1 \mathrm{a}$ & 29,0 a & $30,2 \mathrm{~b}$ \\
\hline Piper nigrum (pimenta do reino) & $6,0 \pm 0,0 \mathrm{~b}$ & $0,2 \pm 0,94 \mathrm{~b}$ & $0,1 \pm 1,0 \mathrm{~b}$ & $100,0 \mathrm{~b}$ & 0,0 * \\
\hline Laurus nobilis (louro) & $0,7 \pm 1,31 \mathrm{a}$ & $54,9 \pm 1,19 a$ & $10,6 \pm 1,2 \mathrm{a}$ & $22,5 \mathrm{a}$ & $30,3 \mathrm{~b}$ \\
\hline Chenopodium ambrosioides (maztruz) & $4,6 \pm 0,83 b$ & $2,4 \pm 2,47 b$ & $2,0 \pm 3,2 b$ & $12,3 b$ & $29,4 b$ \\
\hline Piper tuberculatum (pimenta de macaco) & $6,0 \pm 0,0 \mathrm{~b}$ & $0,7 \pm 1,95 b$ & $0,1 \pm 1,0 b$ & $100,0 \mathrm{~b}$ & 0,0 * \\
\hline Tagetes erecta (cravo de defunto) & $1,5 \pm 0,89 a$ & $47,3 \pm 2,18$ a & $10,4 \pm 0,9 a$ & 32,6 a & $30,4 \mathrm{~b}$ \\
\hline Melissa officinalis (erva cidreira) & $0,8 \pm 1,00 a$ & $44,6 \pm 2,59 a$ & $12,6 \pm 1,5 \mathrm{a}$ & $23,3 \mathrm{a}$ & $30,7 \mathrm{~b}$ \\
\hline Testemunha (sem pó) & $0,5 \pm 0,70 \mathrm{a}$ & $49,3 \pm 3,16 a$ & $10,4 \pm 1,4 \mathrm{a}$ & 29,6 a & $31,8 a$ \\
\hline $\mathrm{H}$ & 75,66 & 72,67 & 67,07 & 40,04 & 28,12 \\
\hline
\end{tabular}

Médias seguidas de mesma letra nas colunas não diferem estatisticamente entre si pelo teste SNK a 5\% de probabilidade.

* Onde não houve emergência, os dados não entraram na análise estatística.

** Período médio de desenvolvimento 
Resultados semelhantes aos desta pesquisa em relação ao cravo da índia, foram encontrados por Paranhos et al. (2005), onde observaram que o uso do cravo da índia se igualou ao uso do inseticida comercial Gastoxin ${ }^{\circledR}$. Neste mesmo trabalho os autores recomendaram a dose de $25 \mathrm{~g}$ de pó de cravo da índia por quilo de feijão, sugerindo ainda pesquisas com doses mais baixas. Segundo Affonso et al. (2012), esta espécie de planta é bastante estudada e possui patentes de produtos para uso diversos, sendo a principal substância estudada o eugenol, provavelmente o princípio ativo causador da repelência e mortalidade dos insetos.

Com relação ao matruz, Procópio et al. (2003b), obtiveram mortalidades de $100 \%$ ao quinto dia, tanto para $Z$. subfasciatus como para Acanthoscelides obtectus. Mazzonetto \& Vendramim (2003) avaliaram o efeito de plantas de mastruz coletadas em locais distintos sobre $A$. obtectus e observaram variação na mortalidade dos insetos de 14 a $100 \%$. Desta forma, podemos sugerir que haja influencia local sobre o nível da atividade inseticida de $C$. ambrosioides. O efeito desta planta sobre $Z$. subfasciatus e A. obtectus tem variado. Baldin et al. (2009) observaram que o pó de matruz estimulou a oviposição de $A$. obtectus, sendo o tratamento com maior número de insetos emergidos e neutro em relação à repelência, efeitos contrário ao observados por Mazzonetto \& Vendramim (2003), e no presente trabalho.

Em relação à postura e emergência, nos grãos tratados com cravo da índia, pimenta de macaco e pimenta do reino, praticamente não houve oviposição e emergência, fato explicado pela morte dos insetos quando em contato com os pós. Em relação ao matruz, apesar da igualdade estatística com os tratamentos relatados acima, ainda houve uma pequena postura; 24 ovos no total, gerando 17 adultos emergidos com 29,4 dias de desenvolvimento médio (Tabela 1). O restante dos pós vegetais avaliados não diferiram da testemunha em postura, emergência e morte prematura. Estes resultados se assemelham aos de Procópio et al. (2003) e Mazzonetto \& Vendramim (2003) onde, os tratamentos que não obtiveram ótima mortalidade de adultos, nos outros parâmetros se igualaram à testemunha. Dessa forma, fica aparente que o impacto inseticida dos materiais testados deve ser na mortalidade dos insetos adultos, já que aparentemente não há efeito ovicida ou larvicida para o inseto em questão, tendo em vista que o padrão de cada tratamento se mantem nos parâmetros ovos viáveis, percentual de emergência e percentual de morte prematura. O contrário ocorreu com Baldin et al. (2009), onde os mesmos acreditam ter ocorrido interação dos pós testados em relação à viabilidade de ovos e ou larvas de A. obtectus.

Em relação ao PMD, houve diferenças estatísticas significativas, porém não podendo ser atribuídas somente às propriedades anti nutricionais dos pós. C. nardus se igualou à testemunha, com os maiores PMD, diferindo das demais.

Pôde-se agrupar, de acordo com o índice de repelência adotado, os pós vegetais em dois grupos; repelente, representado por citronela, cravo da índia e mastruz, e os neutros; pimenta do reino, erva cidreira, pimenta de macaco, e cravo de defunto.

Embora classificada como neutro, observouse que o pó de erva cidreira tende a atrair mais adultos de $Z$. subfasciatus, podendo apresentar uma certa atratividade não detectada pelo índice adotado. Da mesma forma, observou-se que os pós de pimenta de macaco e cravo de defunto atraíram uma quantidade significativamente menor de insetos em relação à testemunha, mas não o suficiente para serem considerados como repelente (Tabela 2 ).

Tabela 2. Repelência de pós vegetais sobre $Z$. subfasciatus em teste com livre chance de escolha.

\begin{tabular}{|c|c|c|c|}
\hline Tratamentos & Insetos Atraídos ( $\pm \mathrm{EP}$ ) & $\begin{array}{c}\text { Índice de Repelência ( } \mathbf{} \\
\text { EP) }\end{array}$ & Classificação \\
\hline Cymbopogon nardus (citronela) & $1,9 \pm 0.89 c$ & $0,3+-0,5$ & Repelente \\
\hline Syzygium aromaticum (cravo da índia) & $0,3 \pm 0,91 \mathrm{c}$ & $0,0+-0,3$ & Repelente \\
\hline Testemunha (sem pó) & $13,9 \pm 1,73 a$ & $1,0+-0,0$ & Neutro \\
\hline Piper nigrum (pimenta do reino) & $14,1 \pm 1,58 \mathrm{a}$ & $1,0+-0,4$ & Neutro \\
\hline Melissa officinalis (erva cidreira) & $21,7 \pm 1,00 \mathrm{a}$ & $1,2+-0,2$ & Neutro \\
\hline Chenopodium ambrosioides (matruz) & $3,1 \pm 1,40 b$ & $0,4+-0,4$ & Repelente \\
\hline Piper tuberculatum (pimenta de macaco) & $9,4 \pm 1,08 b$ & $0,8+-0,4$ & Neutro \\
\hline Tagetes erecta (cravo de defunto) & $9,4 \pm 1,49 b$ & $0,8+-0,4$ & Neutro \\
\hline $\mathrm{H}$ & 44,05 & & \\
\hline
\end{tabular}

Rev. Bras. PI. Med., Campinas, v.16, n.3, p.499-504, 2014. 
Em relação à utilização do pó de citronela, podemos observar que esta planta é altamente repelente à Z. subfasciatus mas não causa alteração nos parâmetros biológicos testados.

França et al. (2012) observaram resultados contrários utilizando esta planta sob a forma de óleo. Estes autores observaram que óleo de $C$. nardus causou $100 \%$ de mortalidade em $Z$. subfasciatus, mas não foi eficiente na repelência deste inseto sendo classificado como neutro. Lima- Mendonça et al. (2013) obtiveram resultados semelhantes aos desta pesquisa em relação à repelência de citronela e matruz sobre Sitophilus zeamais, sobretudo na letalidade da citronela, no entanto, diferindo um pouco em relação à letalidade do mastruz. Segundo Shasany et al. (2000), as substâncias responsáveis pelo efeito repelente desta espécie são monoterpenos voláteis, tais como citronelal, geraniol e eugenol. Este último, já discutido acima, presente também em grandes quantidades em cravo da índia, comprovou a eficácia desta substância na repelência e mortalidade de $Z$. subfasciatus. Dessa forma, sugere-se mais pesquisas em relação à forma de utilização de citronela no controle de $Z$. subfasciatus.

\section{CONCLUSÕES}

P. nigrum (pimenta do reino), P. tuberculatum (pimenta de macaco), S. aromaticum (cravo da índia) e C. ambrosioides (mastruz), são tóxicas à Z. subfasciatus com ação inseticida.

Ruta graveolens (arruda), L. nobilis (louro), T. erecta (cravo de defunto) e C. nardus (citronela), não apresentaram efeito tóxico sobre $Z$. subfasciatus nos parâmetros estudados em teste de confinamento.

C. nardus, S. aromaticum e C. ambrosioides são repelentes à $Z$. subfasciatus, de acordo com o índice de repelência calculado.

$P$. tuberculatum, $P$. nigrum e $T$. erecta são neutras em relação à $Z$. subfasciatus.

$M$. officinalis não tem efeito inseticida nem repelente sobre $Z$. subfasciatus.

$S$. aromaticum e $C$. ambrosioides têm efeito inseticida e repelente sobre $Z$. subfasciatus.

\section{REFERÊNCIA}

AFFONSO, et al. Aspectos Químicos e Biológicos do Óleo Essencial de Cravo da Índia. Revista Virtual de Química, 4(2), 146-161, 2012.

ALMEIDA, et al. Atividade inseticida de extratos vegetais sobre Callosobruchus maculatus (Fabr., 1775) (Coleoptera: Bruchidae). Revista brasileira de Agrociência, 10(1), 67-70, 2004.

BALDIN, et al. Uso de pós de origem vegetal no controle de Acanthoscelides obtectus Say. (Coleoptera: Bruchidae) em grãos de feijoeiro. BioAssay, 4(2), 2009.

BECKEL, H. et al. Detecção da resistência de Oryzaephilus surinamensis (L.) (Coleoptera: Silvanidae), praga de grãos de cevada armazenada, a inseticidas químicos, In: REUNIÃO ANUAL DE PESQUISA DE CEVADA. EMBRAPA TRIGO, 22., 2002, Passo Fundo, RS. Anais. Passo Fundo: 2002. p.620-630.

$\mathrm{BECKEL}, \mathrm{H}$. et al. Efeito de repelência do inseticida deltamethrin sobre insetos de raças resistentes e suscetíveis de Rhyzopertha dominica (F.) (Coleoptera, Bostrichidae) em grãos de trigo armazenado. Revista Brasileira de Entomologia [online], 49(4), 572-574, 2005.

CASTRO, et al. Atividade de extrato de Piper tuberculatum Jacq. (Piperaceae) sobre Spodoptera frugiperda. Revista Ciência Agronômica, 39(3), 437-442, 2008.

CASTRO, et al. Efeito de pós vegetais sobre oviposição de Callosobruchus maculatus (Fabr.) (Coleoptera: Bruchidae) em feijão-caupí. BioAssay, 5(4), 2010.

FRANÇA, et al. Toxicity and repellency of essential oils to Zabrotes subfasciatus (Boheman) (Coleoptera, Chrysomelidae, Bruchinae) in Phaseolus vulgaris L. Acta Amazonica [online], 42(3), 381-386, 2012.

GARCIA, et al. Eficiência de produtos alternativos no controle Zabrotes subfasciatus, e seus efeitos sobre a qualidade das sementes de Phaseolus vulgaris. Pesquisa Agropecuária Tropical, 30(2), 39-42, 2000.

LIMA-MENDONÇA, et al. Efeito de pós-vegetais sobre Sitophilus zeamais (MOTS., 1855) (COLEOPTERA: CURCULIONIDAE). Arquivos do Instituto Biológico, 80(1), 91-97, 2013.

MAZZONETTO \& VENDRAMIM. Efeito de pós de origem vegetal sobre Acanthoscelides obtectus (Say) (Coleoptera: Bruchidae) em feijão armazenado. Neotropical Entomology, 32(1), 145-149, 2003.

MIRANDA, et al. Potencial inseticida do extrato de Piper tuberculatum (Piperaceae) sobre Alabama argillacea (HUEBNER, 1818) (Lepidoptera: Noctuidae). Revista Brasileira de Oleaginosas e Fibrosas, 6(2), 557-563, 2002.

OLIVEIRA \& VENDRAMIM. Repelência de óleos essenciais e pós vegetais sobre adultos de Zabrotes subfasciatus (Boh.) (Coleoptera: Bruchidae) em sementes de feijoeiro. Anais da Sociedade Entomológica do Brasil, 28(3), 549-555, 1999.

PARANHOS, et al. Extrato de neem e cravo da índia no controle de Zabrotes subfasciatus (Boheman) (Coleoptera: Bruchidae) em sementes de feijão armazenado. Colloquium Agrariae, 1(1), 1-7, 2005.

PERRY, T. et al. The biology of insecticidal activity and resistance. Insect Biochemistry and Molecular Biology, 41(7), 411-422, 2011.

PROCÓPIO, et al. Bioatividade de diversos pós de origem vegetal em relação à Sitophilus zeamais mots. (Coleoptera: Curculionidae). Ciência e Agrotecnologia, 27(6), 1231-1236, 2003 a.

PROCÓPIO, et al. Efeito de pós vegetais sobre Acanthoscelides obtectus (SAY) e Zabrotes subfasciatus (BHO.) (Coleoptera: Bruchidae). Revista Ceres, 50(289), 395-405, 2003 b.

RIBEIRO, et al. Insecticide resistance and synergism in Brazilian populations of Sitophilus zeamais (Coleóptera: 
Curculionidae). Journal of Stored Products Research, 39, 21-31, 2003.

SHASANY, et al. Phenotypic and RAPD diversity among Cymbopogon winterianus Jowitt accessions in relation to Cymbopogon nardus Rendle. Genetic Resources and Crop Evolution, 47(5), 553-559, 2000.
SILVA, et al. Comportamento do gorgulho-do-milho frente às doses de permetrina. Comunicata Scientiae, 4(1), 26-34, 2013.

SOBERÓN, et al. Acción biocida de plantas de Piper tuberculatum Jacq. sobre Diatraea saccharalis (Lepidóptera, Pyralidae). Revista Peruana de Biología, 13(1), 107-122, 2006. 